\title{
INFINITE NODAL NONCOMMUTATIVE JORDAN ALGEBRAS; DIFFERENTIABLY SIMPLE ALGEBRAS
}

\author{
BY \\ D. R. SCRIBNER( $\left.{ }^{1}\right)$
}

\begin{abstract}
The first result is that any differentiably simple algebra of the form $A=F 1+R$, for $R$ a proper ideal, 1 the identity element, and $F$ the base field, must be a subalgebra of a (commutative associative) power series algebra over $F$, and is truncated if the characteristic is not zero. Moreover the algebra $A$ contains the polynomial subalgebra generated by the indeterminates and identity of the power series algebra.

This is used to prove that if $A$ is any simple flexible algebra of the form $A=F 1+R$, $R$ an ideal of $A^{+}$, then $A^{+}$is a subalgebra of a power series algebra and multiplication in $A$ is determined by certain elements $c_{i j}$ in $A$ as in

$$
f g=f \cdot g+\frac{1}{2} \sum \frac{\partial f}{\partial x_{i}} \cdot \frac{\partial g}{\partial x_{j}} \cdot c_{\imath,},
$$

where $c_{i j}=-c_{j 1}$ and ". " is the multiplication in $A^{+}$.

This applies in particular to simple nodal noncommutative Jordan algebras (of characteristic not 2).

These results suggest a method of constructing noncommutative Jordan algebras of the given form. We have done this with the restriction that the $c_{i j}$ lie in $F 1$. The last result is that if $A$ is a finitely generated simple noncommutative algebra of characteristic 0 of this form, then Der $(A)$ is an infinite simple Lie algebra of a known type.
\end{abstract}

I. Introduction. Much has been written about simple nodal noncommutative Jordan algebras which are finite-dimensional. The general purpose of this paper is to drop the assumptions for finite-dimensionality and prove several results analogous to the finite-dimensional results. We will always assume characteristic $\neq 2$.

In particular one has the following theorem due to Kokoris [5]: Let $A$ be a simple nodal noncommutative Jordan algebra, finite-dimensional over $F$. Then $F$ has characteristic $p, A^{+}$is the $p^{n}$-dimensional (commutative) associative algebra $A^{+}=F\left[1, x_{1}, \ldots, x_{n}\right], x_{i}^{p}=0, n \geqq 2$, and multiplication in $A$ is given by

$$
f g=f \cdot g+\sum_{i, j=1}^{n} \frac{\partial f}{\partial x_{i}} \cdot \frac{\partial g}{\partial x_{j}} \cdot c_{i j}, \quad c_{i j}=-c_{j i},
$$

where at least one of the $c_{i j}$ has an inverse.

Received by the editors September 10, 1970.

AMS 1969 subject classifications. Primary 1760, 1720; Secondary 1393.

Key words and phrases. Differentiably simple algebras, derivatives, polynomial algebras, power series algebras, nodal noncommutative Jordan algebras, infinite-dimensional noncommutative Jordan algebras, simple infinite Lie algebras.

(1) This work was supported by a NASA Traineeship while the author was at the University of Iowa, Iowa City, Iowa. 
We prove (Theorem 2) analogously that for $A$ a simple flexible algebra of the form $A=F 1+R, R$ a proper ideal of $A^{+}$, that $A^{+}$is a subalgebra of a formal power series algebra (truncated if the characteristic is $p>2$ ) and that multiplication in $A$ is determined by certain $c_{i j}$ as in Kokoris' theorem.

As a consequence a simple nodal noncommutative Jordan algebra is of characteristic $p>2$ and is a subalgebra of a truncated power series algebra.

As in the finite-dimensional case, the result depends on a classification of differentiably simple (commutative associative) algebras of the form $A=F 1+R, R$ a proper ideal. In the finite-dimensional case, with $R$ nil, it was known [5] that such an $A$ was a finitely generated truncated polynomial algebra $F\left[1, X_{1}, \ldots, X_{n}\right], X_{i}^{p}=0$.

In [1], Block gave a generalized version of this, with a much condensed proof. With the assumptions (i) $A$ commutative associative differentiably simple, (ii) $A / R$ a field, (iii) there exists a nonzero $x$ in $A$ with $R x=0$, he proves $A$ is a finitely generated truncated polynomial algebra. Following the general outline of Block's proof, we prove (Theorem 1 ) if $A$ is a differentiably simple algebra of the form $A=F 1+R$, for $R$ a proper ideal, 1 the identity element, and $F$ the base field, then $A$ must be a subalgebra of a (commutative associative) power series algebra over $F$, and is truncated if the characteristic is not zero.

In $\S I V$ we will consider a special case of the converse to the results of Theorem 2. Explicitly, for $P$ a polynomial algebra, $c_{i j}=-c_{j i}$ arbitrary elements in $F 1, \cdot$ the multiplication in $P$, then (1) above gives a noncommutative Jordan algebra structure on $P$. In [4], Schafer has done this for finite-dimensional algebras. Moreover, he has given a criterion for simplicity of the noncommutative structure and studied simplicity of the Lie algebra ad $(A)^{\prime}$. The same condition for simplicity holds in the case we present. Finally, in case $P$ is a finitely generated algebra of characteristic 0 , we show (Theorem 3 ) that $\operatorname{Der}(A)$ is a simple infinite Lie algebra of a known type.

II. We shall now state Theorem 1 in detail and give the proof.

THEOREM 1. Let $A=F 1+R$ be a differentiably simple algebra over the field $F$ and $R$ a proper ideal of $A$. Then $A$ is commutative and associative and

(i) If the characteristic of $F$ is $p \neq 0, R$ is nil and $B \subseteq A \subseteq B^{*}$, where $B$ is the truncated polynomial algebra $F\left[1, X_{i}: i \in H\right] /\left(X_{i}^{p}\right)$ for some index set $H$ and indeterminates $X_{i}$, and $B^{*}$ is the truncated formal power series algebra in these generators.

(ii) If the characteristic of $F=0, A$ is nil semisimple and $B \subseteq A \subseteq B^{*}$, where now $B$ is the polynomial algebra $F\left[1, X_{i}: i \in H\right]$ for some index set $H$ and indeterminates $X_{i}$ and again $B^{*}$ is the formal power series algebra in these generators.

Proof. We first observe that the algebra $A$ must be commutative and associative. Ravisankar has given a simple proof of this in [3]; although he has stated that his algebras are finite-dimensional, the assumption is not used in his argument. The remainder of the proof is accomplished in a number of steps.

(1) $R^{2} \varsubsetneqq R$ and $\bigcap_{i=1}^{\infty} R^{i}=0$. 
Proof. Let $D$ be arbitrary in $\operatorname{Der}(A)$. Then $R=R^{2}$ implies $D(R) \subseteq R$, a contradiction. Similarly $D\left(R^{k+1}\right) \subseteq R^{k}$ for all $k$ implies $D\left(\bigcap R^{k}\right) \subseteq \bigcap R^{k}$ and $\bigcap R^{k}=0$ because it is a differentiable ideal.

In the following steps we shall refer to the $R$-topology of $A$. As for example in [8], the neighborhoods of 0 are determined by the powers $R^{i}$, the topology is metrizable, and there is the notion of the completion (of any subalgebra) with respect to this topology.

Let $\left\{x_{i}+R^{2}: i \in H\right\}$ be a basis of $R / R^{2}$. We define $B \subseteq A$ as the subalgebra of $A$ generated by $\{1\} \cup\left\{x_{i}: i \in H\right\}$. Then with respect to the induced metric on the subalgebra $B$, we define $B^{*}$ to be the completion of the algebra $B$. $B^{*}$ may be thought of either as the set of limits of Cauchy sequences in $B$ or, equivalently, as the set of formal power series in the $x_{i}, i \in H$. Multiplication in $B^{*}$ is well defined by setting $b c=\lim b_{k} c_{k}$ where $b=\lim b_{k}$ and $c=\lim c_{k}$.

(2) Any $a$ in $A$ is the limit of a Cauchy sequence in $B$, and thus we can consider $B \subseteq A \subseteq B^{*}$.

Proof. From the fact that $\left\{x_{i}+R^{2}: i \in H\right\}$ is a basis of $R / R^{2}$ it follows by induction that $\left\{x_{i_{1}}^{t_{1}} \cdots x_{i_{n}}^{t_{n}}: \sum t_{i}=k\right\}$ spans $R^{k} / R^{k+1}$. Thus

$$
\{1\} \cup\left\{x_{i_{1}}^{t_{1}} \cdots x_{i_{n}}^{t_{n}}: \sum t_{i} \leqq k\right\}
$$

spans $A / R^{k+1}$. In particular, for any $a$ in $A$ and any $k, a=a_{k}+y_{k}$, where $a_{k}$ is a polynomial in the $x_{i}$ 's and 1 , and $y_{k}$ is in $R^{k}$. In addition it follows that $\left\{a_{k}\right\}$ is a Cauchy sequence in this $R$-topology and that $a=\lim a_{k}$. But this means that we may consider $A \subseteq B^{*}$, in the sense that $a \rightarrow \lim a_{k}$, the $a_{k}$ 's considered as elements of $B^{*}$, is an algebra monomorphism.

(3) Let $E$ be the polynomial algebra with identity 1 in the indeterminates $\left\{X_{i}: i \in H\right\}$ and $E^{*}$ the power series algebra in the same indeterminates. Then the maximal ideal $M$ in $E$ generated by the $\left\{X_{i}: i \in H\right\}$ has the property that $\bigcap M^{i}=0$ and $E^{*}$ is the completion of $E$ with respect to the topology induced by the $M^{i}$, $i=1,2, \ldots$ Define $h\left(X_{i}\right)=x_{i}$ and $h(1)=1$ from $\{1\} \cup\left\{X_{i}\right\}$ to $\{1\} \cup\left\{x_{i}\right\}$ in $B$. Since the $X_{i}$ are algebraically independent, this first extends to a unique homomorphism from $E$ onto $B$, and then to a homomorphism from $E^{*}$ onto $B^{*}$.

We define $K=\operatorname{ker} h$. The goal before us is to show that if the characteristic is $p \neq 0$, then $K=\left(X_{i}^{p}\right)^{*}$, the ideal of $E^{*}$ generated by $X_{i}^{p}, i \in H$, and if the characteristic is 0 , then $K=0$.

(4) If $I$ is a differentiable ideal of $B^{*}$, then $I=B^{*}$ or $I \cap A=0$.

Proof. Consider $I \cap A$ in $A$. It is an ideal of $A$. Moreover let $D$ be in $\operatorname{Der}(A)$. For any $a$ in $B^{*}$, define

$$
D^{*}(a)=\lim D\left(a_{k}\right)
$$

where $\left\{a_{k}\right\}$ is a sequence in $B$ with limit $a$. It is straightforward to check that $D^{*}$ is well defined, and that $D^{*}$ is in $\operatorname{Der}\left(B^{*}\right)$. Moreover $\left.D^{*}\right|_{A}=D$, so that $D(I \cap A)$ $\subseteq I \cap A$. Thus $I \cap A$ is a differentiable ideal of $A$ and as such must equal 0 or all 
of $A$. In the latter case 1 is in $A=I \cap A$ and $I=B^{*}$. I.e., either $I \cap A=0$, or $I=B^{*}$.

(5) Let $\Delta=\left\{D^{*} \in \operatorname{Der}\left(E^{*}\right)\right.$ : there exists a $D \in \operatorname{Der}\left(B^{*}\right)$ with $\left.h \circ D^{*}=D \circ h\right\}$. Then (i) $K$ is a $\Delta$-ideal; moreover (ii) if $L \supseteq K$ is a $\Delta$-ideal of $E^{*}$ then either $L=E^{*}$ or $L \cap E \subseteq K$.

Proof. (i) is straightforward.

(ii) First note that if $D$ is in $\operatorname{Der}\left(B^{*}\right)$ with $D\left(x_{i}\right)=a_{i}$ in $B^{*}$, then we can define

$$
D^{*}\left(X_{i}\right)=f_{i}
$$

for any $f_{i}$ in $E^{*}$ such that $h\left(f_{i}\right)=a_{i}$ and extend $D^{*}$ uniquely to a derivation $D^{*}$ on $E^{*}$ which automatically satisfies $h \circ D^{*}=D \circ h$. Namely, we first extend $D^{*}$ to a unique derivation of $E$ by induction. Then for $a=\lim g_{n}$ arbitrary in $E^{*}$, define

$$
D^{*}(a)=\lim D^{*}\left(g_{n}\right) .
$$

Then $D^{*}$ is a well-defined derivation of $E^{*}$; finally because $D \circ h$ and $h \circ D^{*}$ agree on the $X_{k}, k \in H$, the usual procedure shows that they agree on $E^{*}$.

This implies $h(L)$ is a Der $\left(B^{*}\right)$-ideal of $B^{*}$, for given $D$ in $\operatorname{Der}\left(B^{*}\right)$, we have a $D^{*}$ in $\Delta$ with $h \circ D^{*}=D \circ h$, so

$$
D(h(L))=h\left(D^{*}(L)\right) \subseteq h(L) .
$$

But now step (4) implies $h(L) \cap A=0$ or $h(L)=B^{*}$. In case $h(L) \cap A=0$, we obtain $L \cap E \subseteq K$. On the other hand, if $h(L)=B^{*}, 1$ must be in $L$, and therefore $L=E^{*}$.

(6) $\operatorname{Der}\left(E^{*}\right)(K) \cap E \subseteq K$.

Proof. As in [1], $L=\operatorname{Der}\left(E^{*}\right)(K)+K$ is a $\Delta$-ideal of $E^{*}$ which contains $K$. Thus (5) implies $\operatorname{Der}\left(E^{*}\right)(K)+K=E^{*}$ or $\operatorname{Der}\left(E^{*}\right)(K) \cap E \subseteq K$. Now suppose $\operatorname{Der}\left(E^{*}\right)(K)+K=E^{*}$. Then in particular 1 is in $\operatorname{Der}\left(E^{*}\right)(K)+K$. But then $K$ would have to have elements with nonzero terms of degree less than or equal to 1 in the $X_{i}$. Thus there exists a $k=\sum_{n=0}^{\infty} k_{n}$ in $K$ with $k_{n}$ the form of degree $n$ in the $X_{i}$, and with $k_{0}+k_{1} \neq 0$. If we write $k_{0}+k_{1}=\alpha 1+\sum \alpha_{i}, X_{i,}$, with $\alpha, \alpha_{i}$, in $F$, then $h\left(k_{0}+k_{1}\right)=\alpha 1+\sum \alpha_{i}, x_{i,}$. But $h\left(k_{0}+k_{1}\right)=h\left(k-\sum_{n=2}^{\infty} k_{n}\right)=-h\left(\sum_{n=2}^{\infty} k_{n}\right)$, which is in $R^{2}$. However $\alpha 1+\sum \alpha_{i}, x_{i}$ in $R^{2}$ is a contradiction to the fact that the $x_{i}+R^{2}$ were chosen to be a basis of $R / R^{2}$. This completes the proof of (6).

(7) If the characteristic of $F$ is $p \neq 0$, then $K \supseteq\left(X_{i}^{p}\right)$, the ideal of $E$ generated by $X_{i}^{p}, i \in H$.

Proof. Consider $J=\left\{x^{p}: x \in R\right\} . J A \subseteq R A \subseteq R$, so $J A$ is a proper ideal of $A$. Moreover, if $D$ is in $\operatorname{Der}(A), D(J A) \subseteq D(J) A+J D(A) \subseteq J A$, so $J A$ is a proper differentiable ideal of $A$ and therefore $=0$. Thus $J=0$, so in particular $x_{i}^{p}=0$ for all $i$ in $H$. As $h\left(X_{i}\right)=x_{i}$, this implies $\left(X_{i}^{p}\right) \subseteq K$.

(8) If the characteristic is $0, K \cap E=0$. If the characteristic is $p \neq 0$, then $K \cap E$ $=\left(X_{i}^{p}\right)$, the ideal of $E$ generated by the $X_{i}, i \in H$. Consequently the proof of the theorem is completed. 
Proof. By step (6) we know that $\operatorname{Der}\left(E^{*}\right)(K) \cap E \subseteq K$. Suppose there exist polynomials in $K$ and let $f$ be such a polynomial. For any fixed $j \in H, \partial / \partial X_{j}$ is in $\operatorname{Der}\left(E^{*}\right)$ and $\partial / \partial X_{j}: E \rightarrow E$ (as $E$ is just the polynomials of $\left.E^{*}\right)$. Therefore

$$
\partial f / \partial X_{j} \text { is in } \operatorname{Der}\left(E^{*}\right)(K) \cap E \subseteq K \cap E .
$$

Also $\partial f / \partial X_{j}$ is of lesser degree in the $X_{i}$ than $f$. Now suppose

$$
c_{(t)} X_{i_{1}}^{t_{1}} \cdots X_{i_{n}}^{t_{n}}, \quad c_{(t)} \text { in } F,
$$

is a term of $f$ (and there does not exist another term of $f$ of the form $\alpha X_{i_{1}}^{t_{1}} \cdots X_{i_{n}}^{t_{n}}, \alpha$ in $F)$. Then

$$
t_{j} c_{(t)} X_{i_{1}}^{t_{1}} \cdots X_{i_{j}}^{t_{j}-1} \cdots X_{i_{n}}^{t_{n}}
$$

is the only term of $\partial f / \partial X_{j}$ of the form $\alpha X_{i_{1}}^{t_{1}} \cdots X_{i_{j}}^{t_{j}-1} \cdots X_{i_{n}}^{t_{n}}, \alpha$ in $F$. We shall denote the degree 0 form of $f$ by $c_{(0)}$ in the following.

From here on it is less confusing if we consider the characteristic $p=0$ and $p \neq 0$ separately. If the characteristic is 0 we will assume $f$ has been chosen to have minimal degree in the $X_{i}$. By the last paragraph, this forces $\partial f / \partial X_{j}=0$ for any fixed $j$ in $H$. Therefore also $t_{j} c_{(t)}=0$ for all $c_{(t)}$ and any $j$ in $H$. But then if $c_{(t)} \neq 0, t_{j}=0$ must hold for all $j$ in $H$, and $c_{(t)} X_{i_{1}}^{t_{1}} \cdots X_{i_{n}}^{t_{n}}$ must be the degree 0 form of $f$. That is, except for the degree 0 form $c_{(0)}$, all the coefficients $c_{(t)}$ are zero; hence $f=c_{(0)}$. But then $h(f)=h\left(c_{(0)}\right)=c_{(0)}$ must also be zero as it was assumed that $f$ was in $K$. That is, $K$ contains no nonzero polynomials and $h: E \rightarrow B$ is an algebraic isomorphism in this case, which in turn implies $h: E^{*} \rightarrow B^{*}$ is also an algebraic isomorphism.

If the characteristic is $p \neq 0$, we want to show that all polynomials in $K$ must lie in $\left(X_{i}^{p}\right)$. Hence we now assume $f$ is a polynomial of minimal degree in $K$ such that

$f$ is not in $\left(X_{i}^{p}\right)$.

Using the above notation for $f$, we have $\partial f / \partial X_{j}$ is in $\left(X_{i}^{p}\right)$ by the minimality of $f$ not in $\left(X_{i}^{p}\right)$, and so each term

$$
t_{j} c_{(t)} X_{i_{1}}^{t_{1}} \cdots X_{i_{j}}^{t_{j}-1} \cdots X_{i_{n}}^{t_{n}}
$$

of $\partial f / \partial X_{j}$ is in $\left(X_{i}^{p}\right)$. If we have $X_{i_{1}}^{t_{1}} \cdots X_{i_{j}}^{t_{j}-1} \cdots X_{i_{n}}^{t_{n}}$ in $\left(X_{i}^{p}\right)$, then multiplying by $c_{(t)} X_{i,}$ forces $c_{(t)} X_{i_{1}}^{t_{1}} \cdots X_{i_{n}}^{t_{n}}$, the $c_{(t)}$ term of $f$, to lie in $\left(X_{i}^{p}\right)$. On the other hand, if $X_{i_{1}}^{t_{1}} \cdots X_{i_{j}}^{t_{j}-1} \cdots X_{i_{n}}^{t_{n}}$ is not in $\left(X_{i}^{p}\right)$, we must have $t_{j} c_{(t)}=0$. As we can assume $c_{(t)} \neq 0$, this implies

$$
t_{j} \equiv 0 \quad(\bmod p) .
$$

If $t_{j}$ is positive and divisible by $p$, clearly the $c_{(t)}$ term of $f$ lies in $\left(X_{i}^{p}\right)$. The only remaining case is when $t_{j}=0$ for all $j$ in $H$ and $c_{(t)}$ is the degree 0 form, $c_{(0)}$, of $f$. Altogether this implies that $f \equiv c_{(0)}\left(\bmod \left(X_{i}^{p}\right)\right)$. But in step (7) we showed that $\left(X_{i}^{p}\right) \subseteq K$, and so this last equation implies that $c_{(0)}$ is in $K$. This is a contradiction unless $c_{(0)}=0$, in which case $f$ is in $\left(X_{i}^{p}\right)$, again a contradiction to the choice of $f$. This completes the proof that $E \cap K \subseteq\left(X_{i}^{p}\right)$. Since step (7) gives us the other containment we have the equality $E \cap K=\left(X_{i}^{p}\right)$ as desired. 
Thus finally, we have that $h$ induces an algebraic isomorphism from $E /\left(X_{i}^{p}\right)$ onto $B$, which in turn implies that $E^{*} /\left(X_{i}^{p}\right)^{*}$ and $B^{*}$ are algebraically isomorphic, where $\left(X_{i}^{p}\right)^{*}$ denotes the ideal of $E^{*}$ generated by $\left(X_{i}^{p}\right)$. This completes the proof of the theorem.

III. We now apply Theorem 1 to simple flexible algebras of the form $A=F 1+R$ for $R$ a proper ideal of $A^{+}$. The proof of the following lemma may be found in [5].

LEMMA. If $A$ is a simple flexible algebra of the form $A=F 1+R$, for $R$ a proper ideal of $\mathrm{A}^{+}$, then $\mathrm{A}^{+}$is differentiably simple.

THEOREM 2. Let A be a simple flexible algebra of degree 1 over a field $F$ of characteristic $\neq 2$, and suppose $A$ is of the form $F 1+R$ for $R$ a proper nonzero ideal of $A^{+}$. Then $A^{+}$is a subalgebra of a (truncated if characteristic $>2$ ) power series algebra as described in Theorem 1, and multiplication in $A$ is given by

$$
f g=f \cdot g+\frac{1}{2} \sum \frac{\partial f}{\partial x_{i}} \cdot \frac{\partial g}{\partial x_{j}} \cdot c_{i j}, \quad c_{i j} \text { in } A,
$$

where $c_{i j}=-c_{j i}$ and at least one $c_{i j}$ is not in $R$.

Proof. The first statement follows by Theorem 1 and the above lemma. Now let $f$ be in $A$. That $[f]=,\operatorname{ad} f$ is a derivation of $A^{+}$is well known [5]. Suppose $\left[f, x_{j}\right]$ $=a_{j}(f)$ in $A$. Then if $g$ is any polynomial in $A$, it is clear by induction that $[f, g]$ $=\sum\left(\partial g / \partial x_{j}\right) \cdot a_{j}(f)$. If $h$ in $A$ is not a polynomial, suppose $g_{n}$ is a sequence of polynomials in $A$ converging to $h$. For any $D$ in $\operatorname{Der}\left(A^{+}\right), D\left(\lim g_{n}\right)=\lim D\left(g_{n}\right)$. In particular, $[f, h]$, and $\partial / \partial x_{j}$ are in $\operatorname{Der}\left(A^{+}\right)$, so we have

$$
[f, h]=\lim \left[f, g_{n}\right]=\lim \sum \frac{\partial g_{n}}{\partial x_{j}} \cdot a_{j}(f)=\sum \frac{\partial h}{\partial x_{j}} \cdot a_{j}(f) .
$$

Next, by the same argument the derivation $\left[x_{j},\right]$ is determined by the $a_{i}\left(x_{j}\right)$ $=\left[x_{j}, x_{i}\right]$, so

$$
\left[x_{j}, f\right]=\sum \frac{\partial f}{\partial x_{i}} \cdot\left[x_{j}, x_{i}\right]
$$

But $\left[x_{j}, f\right]=-\left[f, x_{j}\right]=-a_{j}(f)$ then implies

$$
-a_{j}(f)=\sum \frac{\partial f}{\partial x_{i}} \cdot\left[x_{j}, x_{i}\right] .
$$

Substituting in our last calculation of $[f, h]$, we obtain

$$
\begin{aligned}
{[f, h] } & =\sum \frac{\partial h}{\partial x_{j}} \cdot a_{j}(f) \\
& =-\sum \frac{\partial h}{\partial x_{j}} \cdot \frac{\partial f}{\partial x_{i}} \cdot\left[x_{j}, x_{i}\right] \\
& =\sum \frac{\partial f}{\partial x_{i}} \cdot \frac{\partial h}{\partial x_{j}} \cdot\left[x_{i}, x_{j}\right] .
\end{aligned}
$$


By setting $c_{i j}=\left[x_{i}, x_{j}\right]$ and noting that $f h=f \cdot h+\frac{1}{2}[f, h]$, we have the multiplication determined as desired. Finally note that if all $c_{i j}$ were in $R, R$ would be an ideal of $A$, a contradiction.

COROLlARY. If $A$ is a simple nodal noncommutative Jordan algebra of characteristic not 2, then A satisfies the hypothesis and hence the conclusions of Theorem 2. (Thus the characteristic is necessarily $p>2$.)

Proof. If $R=$ the set of nilpotent elements, McCrimmon's result [2] implies $R$ is an ideal of $A^{+}$.

IV. In light of Theorem 2 it seems logical to construct noncommutative Jordan algebras by introducing new multiplications on subalgebras of power series algebras. Although this can be done quite generally, our result here deals with the following special case. Let $P$ be a polynomial algebra $F\left[1, x_{i} \in H\right], x_{i}^{p}=0$ if the characteristic of $F$ is greater than 2, and suppose $c_{i j}=-c_{j i}$ are arbitrary elements in $F$. Then define $A$ as the vector space of $P$ with the multiplication

$$
f g=f \cdot g+\frac{1}{2} \sum \frac{\partial f}{\partial x_{i}} \cdot \frac{\partial g}{\partial x_{j}} \cdot c_{i j} \quad \text { ("." the } P \text {-multiplication). }
$$

An easy computation shows that $A$ is flexible and hence a noncommutative Jordan algebra. Suppose the bilinear form induced by $\left(x_{i}, x_{j}\right) \rightarrow c_{i j}$ on the subspace $V$ of $A$ with basis $\left\{x_{i}: i \in H\right\}$ is nondegenerate. Then assuming $A$ not simple choose $f$ to be a nonzero element in a nontrivial ideal $I$ of $A$. Without loss of generality we may also suppose $f$ involves only the generators $x_{1}, \ldots, x_{n}$. Since $($,$) is non-$ degenerate there exists a $y$ in $V$ such that $\left(y, x_{i}\right)=0$ for $i=1, \ldots, n-1$, and $\left(y, x_{n}\right)=1$. That is, $y$ satisfies $\left[y, x_{i}\right]=0$ for $i=1, \ldots, n-1$, and $\left[y, x_{n}\right]=1$. However the ideal $I$ must contain $y f-f y=[y, f]$. As $[y$,$] is a derivation of A^{+}$, we have $[y, f]=\sum\left[y, x_{i}\right] \cdot \partial f / \partial x_{i}=\partial f / \partial x_{n}$. This process leads to a nonzero element in $I$ which is void in $x_{n}$ and involves only $x_{1}, \ldots, x_{n-1}$, which in turn leads to a nonzero element in $I$ which is a constant, contrary to the choice of $I$. That is, (, ) nondegenerate implies $A$ is simple.

Conversely, if $($,$) is degenerate, there is an x$ in $V$ such that $(x, y)=0$ for all $y$ in $V$; in particular $\left(x, x_{i}\right)=\left[x, x_{i}\right]=0$ for $i$ in $H$. But then by induction we obtain $[x, f]=0$ for any $f$ in $A$. Finally $[x, A]=0$ implies that $x \cdot A$ is a nonzero proper ideal of $A$. Thus degeneracy of ( , ) implies that $A$ is not simple.

We now restrict $A$ to be a finitely generated simple polynomial algebra (with the $c_{i j} \in F$ multiplication) and study the derivations of $A$. Since a finitely generated algebra of this type of characteristic $p>2$ is also finite-dimensional, and this case is well known [4], we shall moreover expressly assume $F$ is of characteristic 0 . By the above two paragraphs there are an even number of generators, say $x_{1}, \ldots, x_{2 n}$. We shall assume $\left[x_{2 i-1}, x_{2 i}\right]=1$ for $i=1, \ldots, n$, and $\left[x_{k}, x_{j}\right]=0$ if $\{k, j\} \neq\{2 i-1,2 i\}$ for all $i$. Now $D$ in $\operatorname{Der}(A)$ implies $D$ is in $\operatorname{Der}\left(A^{+}\right)$and it is clear that any derivation of $A^{+}$is completely determined by where the generators are mapped, i.e., $D\left(x_{i}\right)=a_{i}$ 
in $A$ determines $D$ (and conversely $x_{i} \rightarrow a_{i}$, for $a_{i}$ arbitrary elements in $A$, induces a unique derivation of $A^{+}$). Under these conditions, Schafer has shown in [4] that for $D$ a derivation of $A^{+}, D$ is in $\operatorname{Der}(A)$ if and only if

$$
\begin{aligned}
& \frac{\partial a_{i}}{\partial x_{j+1}}=\frac{\partial a_{j}}{\partial x_{i+1}} \text { for } i, j \text { odd. } \\
& \frac{\partial a_{i+1}}{\partial x_{j}}=\frac{\partial a_{j+1}}{\partial x_{i}} \quad \text { for } i, j \text { odd. } \\
& \frac{\partial a_{i}}{\partial x_{j}}+\frac{\partial a_{j+1}}{\partial x_{i+1}}=0 \text { for } i, j \text { odd. }
\end{aligned}
$$

THEOREM 3. Let $A$ be a simple noncommutative Jordan algebra over the field $F$ of characteristic $0, A^{+}=F\left[1, x_{1}, \ldots, x_{2 r}\right]$, and multiplication in $A$ be determined by $c_{2 k-1,2 k}=-c_{2 k, 2 k-1}=1$ for $k=1,2, \ldots, r$, and $c_{i, j}=0$ if $\{i, j\} \neq\{2 k-1,2 k\}$ for all $k$. Then $\operatorname{Der}(A)$ is a simple Lie algebra of a known type.

Proof. We refer to R. L. Wilson's notation and remarks in [7]. There $C=$ Complex numbers, $\mathscr{W}(m)=\operatorname{Der} C\left[\left[x_{1}, \ldots, x_{m}\right]\right]$ and

$$
\mathscr{V}(2 r)=\left\{D \in \mathscr{W}(2 r): D w=0, w=\sum_{i=1}^{r} d x_{2 i-1} \wedge d x_{2 i}\right\}
$$

(As is there explained, "the action of a derivation $D$ on the algebra $\mathscr{D}$ of differential forms is that of the Lie derivative. Thus $\mathscr{D}$ is the exterior algebra on $\left\{d x_{1}, \ldots, d x_{m}\right\}$ over $C\left[\left[x_{1}, \ldots, x_{m}\right]\right], d f=\sum\left(\partial f / \partial x_{i}\right) d x_{i}, D(d f)=d(D f), D(f w)=(D f) w+f(D w)$, and $D(w \wedge z)=D w \wedge z+w \wedge D z$ for all $f \in C\left[\left[x_{1}, \ldots, x_{m}\right]\right]$ and all $w$ and $z$ in $\left.\mathscr{D} . "\right)$

Now it is clear that the assumptions and conclusions are unchanged if one assumes $F=C$. Then it is a straightforward calculation that Schafer's conditions (1)-(3) for $D \in \operatorname{Der}(A)$ are equivalent to $\operatorname{Der}(A)=\mathscr{V}(2 r)$. But $\mathscr{V}(2 r)$ is a simple infinite Lie algebra of Cartan type over $C$ [7].

A simple corollary to this result is that ad $(A)$, which must equal $\operatorname{Der}(A)$, is also a simple Lie algebra. There is a possibility that if the $c_{i j}$ were not restricted to $F 1$, but $A$ were assumed to be Lie admissible, simple, and of characteristic 0 , that ad $(A)$ would still be simple. We have not resolved this, but we have shown the derived algebra ad $(A)^{\prime}$ is simple under the assumption that $A$ is simple and Lie admissible [6].

\section{BIBLIOGRAPHY}

1. Richard E. Block, Determination of the differentiably simple rings with a minimal ideal (to be published).

2. Kevin McCrimmon, Jordan algebras of degree 1, Bull. Amer. Math. Soc. 70 (1964), 702. MR 29 \#2286.

3. T. S. Ravisankar, A note on a theorem of Kokoris, Proc. Amer. Math. Soc. 21 (1969), 355356. MR 39 \#271. 
4. R. D. Schafer, Nodal noncommutative Jordan algebras and simple Lie algebras of characteristic p, Trans. Amer. Math. Soc. 94 (1960), 310-326. MR 22 \#8044.

5. — An introduction to nonassociative algebras, Pure and Appl. Math., vol. 22, Academic Press, New York, 1966. MR 35 \#1643.

6. D. R. Scribner, Lie-admissible, nodal, noncommutative Jordan algebras, Trans. Amer. Math. Soc. 154 (1971), 105-111.

7. R. L. Wilson, Nonclassical simple Lie algebras, Bull. Amer. Math. Soc. 75 (1969), 987-991.

8. Oscar Zariski and Pierre Samuel, Commutative algebra. Vol. 2, University Series in Higher Math., Van Nostrand, Princeton, N. J., 1960. MR 22 \#11006.

UNIVERSITY OF GEORGIA,

Athens, GeORgia 30601 